\title{
Remote monitoring system for embedded network equipment faults based on Internet of Things
}

\author{
Wei-xue LIU \\ \{tiantiankaixin255@tom.com\} \\ (Collage of Mathematics and Compute Science,Chifeng University ,Chifeng 024000,china)
}

\begin{abstract}
The current remote monitoring system for network equipment faults mainly uses three technologies: web, Internert, and CAN. Although these three systems meet the needs of the monitoring scope.However, when collecting data, due to insufficient information and simple analysis of the data, the accuracy of system monitoring is poor.To this end, a remote monitoring system for embedded network equipment faults based on the Internet of things is studied.Based on the requirements analysis and combined with the industrial Internet of Things technology, the overall scheme of the system is given, and the system hardware design is carried out from the three aspects of the collector, communication equipment and host, and the corresponding software is designed in detail based on the three main system hardware. The results show that compared with the three remote monitoring systems for network equipment faults based on web, Internert, and CAN, the system has higher monitoring accuracy while ensuring the monitoring range, which indicates that the system has stronger comprehensive monitoring performance.
\end{abstract}

Key words: Internet of things; Internet equipment; Fault monitoring; system design

\section{Introduction}

With the rapid development of social science and technology, research and innovation in various fields are constantly emerging, and knowledge has grown rapidly in the form of explosion.Some of the main characteristics of the information age are networking, digitalization, and informationization. Because the characteristics of the network can transmit information very quickly, the realization of informatization must rely on a perfect network [1]. The Internet has now developed into the lifeblood of today's information society and a key foundation for the development of the knowledge economy, and has had a significant impact on all aspects of social life, especially social and economic development. Since the 1990s, computer networks, typified by the Internet, have developed rapidly. From the initial scientific research and education network to a commercial network, it has gradually changed all aspects of our work and life and accelerated global informationization. The process of the revolution, 
now people's work, life, study and communication can not leave the Internet.In this context, more and more devices are connected to the network, and the types of network devices are increasing. The current basic network devices are: computers, hubs, switches, bridges, routers, gateways, network interface cards (NICs), wireless Access points (WAP), printers and modems, fiber optic transceivers, fiber optic cables, etc., so failures are unavoidable, which brings a lot of inconvenience to people's lives and work [2]. Network equipment failure monitoring is of great significance to the long-term normal operation of communication systems, and to social production and life, as well as national defense security.In some special environments such as military weapon systems, the reliability of network systems is particularly important. Especially during the execution of certain critical tasks, the occurrence of network failures may lead to the loss of important information and even cause mission failure.Fault prediction for network equipment is of great significance for improving the reliability of network systems, ensuring the smooth execution of key tasks, and reducing the burden of network management and maintenance.

Currently, network equipment fault monitoring systems mainly include three types of network equipment fault remote monitoring systems based on web, Internert, and CAN However, although the monitoring scope of these three systems meets requirements, the monitoring performance is insufficient. Aiming at the above problems, a technical solution of the remote monitoring system for embedded network equipment faults based on the Internet of things is proposed.In the absence of monitoring, notify the network administrator of the faulty device name and other information in the form of a mobile phone message in real time, so that the network administrator can detect the network fault in a timely manner and repair the fault in a short time to ensure network smoothness and data security [3]. The test results show that: compared with the fault remote monitoring system based on web, Internert and CAN, the system has better monitoring performance, a wider monitoring range, and has reached the expected goals of the research.

\section{Design of remote monitoring system for embedded network equipment faults}

With the rapid development of the current Internet of Things technology, related equipment has entered the era of the Internet of things, making the application rate of different intelligent IoT machinery and equipment continue to increase. The normal operation of the network center equipment is the basic condition to ensure the unit's Internet of things access. Sudden failures in the network equipment during operation will cause great losses if it is not repaired in time. Therefore, effective methods are sought to ensure the normality of the equipment Operation has become a hotspot for related personnel analysis [4]. For this reason, 
designing a reasonable intelligent IoT equipment fault monitoring system has high application significance.

\section{1 system framework}

Industrial Internet of Things technology can help enterprises to establish a complete network architecture and realize the monitoring and management of the entire production process of equipment. At present, the industrial Internet of things technology is relatively mature, and there are successful cases in other industries. Therefore, it is feasible to apply the Industrial Internet of things technology to the equipment digital monitoring industry [5]. The Industrial Internet of things model is divided into four levels. This section divides the hierarchical structure of the equipment monitoring management system based on the industrial Internet of things model and system requirements. It is divided into physical layer, interconnection layer, network layer, data layer, service layer, application layer, as shown in Figure 1.

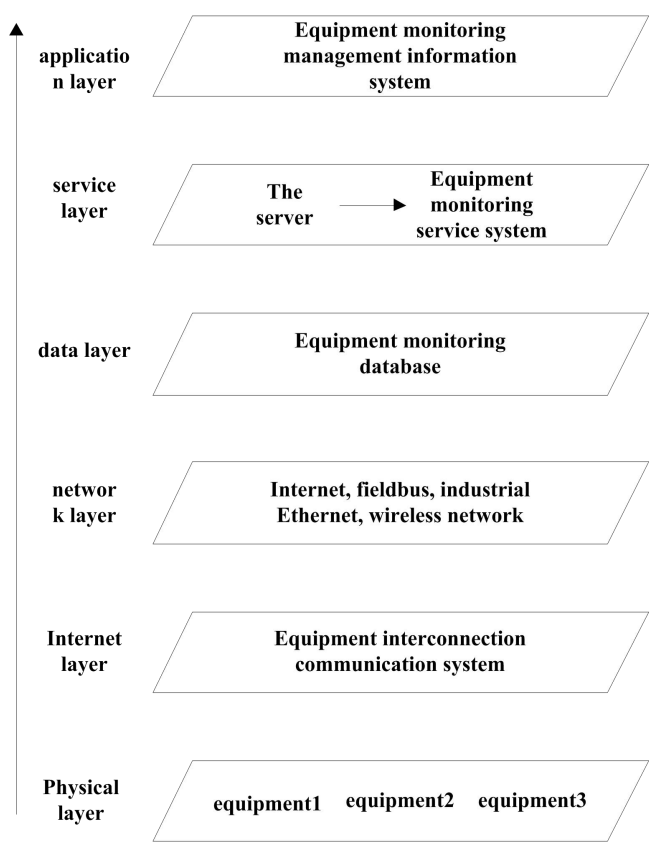

Fig.1 IoT technology hierarchy

Physical layer: Mainly refers to the underlying physical equipment and perception system. During the operation of network equipment, its operating data will be stored by the equipment's PLC, so the physical equipment and sensing system here mainly refers to the PLC of the machine tool equipment [6].

Interconnection layer: It mainly refers to the interconnection communication system of 
network equipment. It is responsible for the interconnection between equipment and the interconnection between the equipment and the network layer, and collects the equipment operation data in the equipment PLC, and sends these data to the network layer.

Network layer: It refers to the network equipment monitoring network system, which is a communication bridge between the systems, corresponding to various network systems in the production environment. In the factory, the production data collected by the interconnection layer needs to be passed upwards, and at the same time, the operation instructions of each device need to be issued to the interconnection layer. Due to the strong functionality of industrial Ethernet, it is suitable for various complex environments. Industrial Ethernet is mainly used as a device monitoring network system [7].

Data layer: Mainly refers to the network equipment monitoring database to realize the classified storage of real-time operating data of the equipment.

Service layer: Refers to the network equipment monitoring service system, which summarizes and organizes equipment operation data to meet the requirements of enterprises to implement digital monitoring.

Application layer: Network equipment monitoring and management information system, through the analysis of network equipment operation data, the overall control and optimization of operation.

Based on the analysis of the composition of the Internet of things technology, the architecture of the device monitoring and management system is designed. As shown in Figure 2 , it is mainly divided into four levels: the device interconnection acquisition layer, the network transmission layer, the data storage layer, and the application service layer.

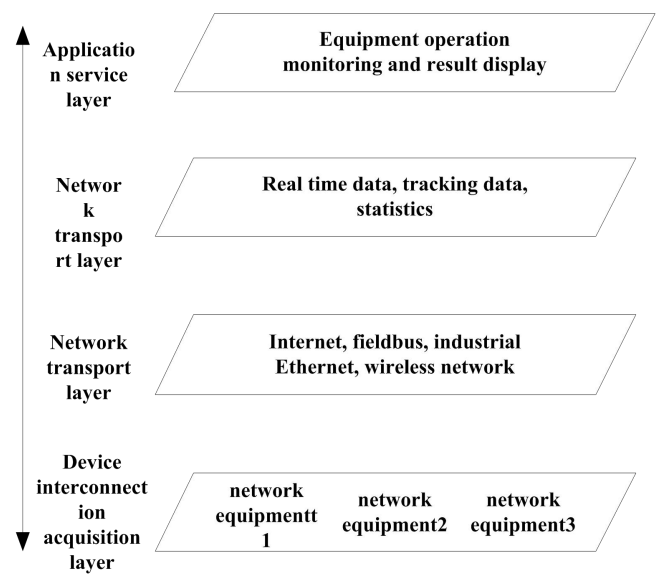

Fig.2 System framework hierarchy

\subsection{System hardware}


In terms of physical layout, the system adopts a split design, which is divided into a host and a collector. This design can prevent the signal from being shielded when the system uses wireless transmission. The host implements the core functions of the front-end monitoring system, which can be installed in network equipment, and the collector is responsible for data collection [8]. The host and the collector can use GPRS communication. The hardware module diagram of the front-end monitoring system is shown in Figure 3.

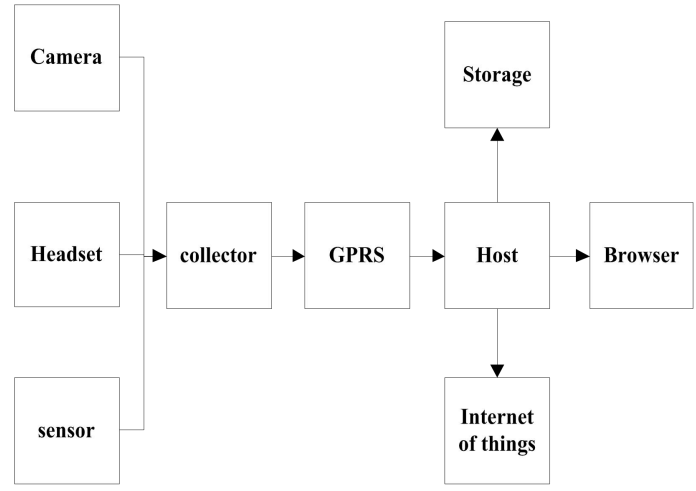

Fig.3 Hardware module diagram of the front-end monitoring system

\section{(1) Collector}

In this paper, the system data acquisition module is mainly responsible for collecting real-time parameters of the faulty equipment in the IoT environment, and detecting the system status of the faulty equipment.

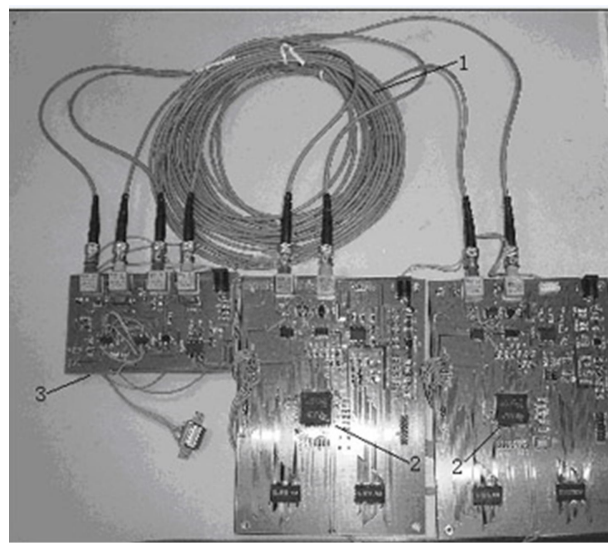

( 1 is communication line, 2 is LF2407A chip, 3 is converter)

Fig.4 Circuit physical diagram of the data acquisition module

The data acquisition module mainly includes sensors, power adapters, wireless communication modules, equipment fault data storage modules, A / D converters and communication interfaces. The sensor is used to collect the data of the faulty device, the 
power adapter provides energy for the overall data acquisition module, and the wireless communication module implements the wireless transmission of data through the IEEE 802.15.4 protocol. The data acquisition module performs operations such as filtering, amplifying, sampling / holding, and A / D conversion on the signals sent from the field of the faulty device through sensors, and stores the data of the faulty device in the device fault data storage module. Device information is passed between modules [9].

In order to ensure the utilization of the server, generally one acquisition device is connected to 3-5 network devices for acquisition, and the devices are mainly distinguished according to the IP number and port number set in the device to achieve the correspondence between the device and the operating data. In order to ensure the reliability of data collection and upload of the equipment in some unexpected situations, a backup server is configured for each server. When the collection server fails to work normally, the system will automatically call the backup server for jobs.

(2) GPRS communication equipment

The system of this paper realizes the efficient transmission of faulty device data in the Internet of things through the communication network module. The communication network module is the bridge of the overall system and the link between the data acquisition module and the detection and diagnosis module. It plays an important role. The communication module is mainly composed of a SIM900B controller, a radio frequency module, a power module, a memory, and an application interface. Its advantages are: providing voice, data, SMS fax and other services; built-in TCP / IP protocol stack; low power consumption, only 2.0ma current in sleep state. The core controller of the GPRS terminal communication module comes from SIMCom's wireless quad-band SIM900B controller, which can send data information with the lowest power consumption, as shown in Figure 5.

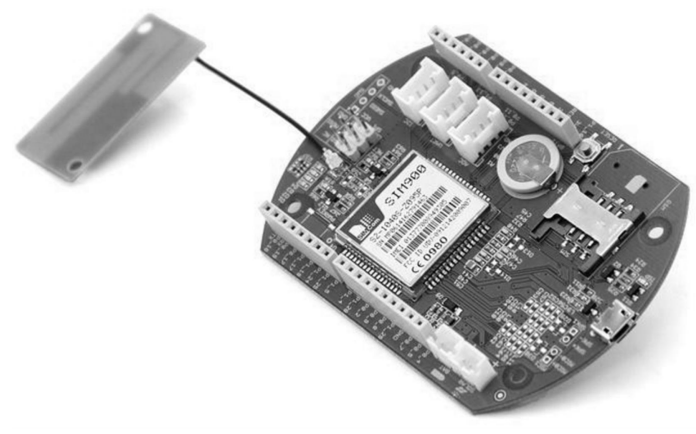

Fig.5 M900B controller

SIM900B controller characteristic parameters are shown in Table 1. 
Table 1 SIM900B controller characteristic parameters

\begin{tabular}{cc}
\hline Characteristic & Explain \\
\hline Dual frequency & Support both GSM and GPRS, working frequency $850 / 900 /$ \\
& $1800 \mathrm{MHz}$ \\
GPRS rate & GPRS downlink transmission, maximum $85.6 \mathrm{kbps}$ \\
Serial port & Standard serial port, transmission rate $1.2 \mathrm{k}-115.2 \mathrm{kbps}$ \\
Interface & Support mobile and Unicom SIM card \\
control & At command control \\
function & Telephone, SMS, GPRS communication, MMS, base station \\
& positioning, audio \\
power supply & $3.1-4.8 \mathrm{VDC}$ \\
working temperature & $-40-85^{\circ} \mathrm{C}$ \\
\hline
\end{tabular}

(3) Host

The host is at the center of the entire monitoring system, and maintains a close relationship with the sensor nodes. It can interact with the user through a WEB browser. The host can receive the data collected by the sensor and save the data in the host's database. At the same time, the host can provide a communication interface based on the HTTP protocol. The user can send control instructions to the host through these interfaces. It can query the corresponding data stored in the ECS database according to the instructions, or feed back to the sensor node according to the instructions sent by the web end, so as to control the sensor node of the lower computer.

In this article's remote monitoring system, we adopted the cloud Elastic Compute Service (ECS) and Open Storage Service (OSS) provided by Alibaba Cloud to jointly constitute the host used by the monitoring system.The ECS cloud server handles storing data, and OSS provides auxiliary storage space, which can be customized according to user needs. Figure 6 below shows the structure of the host system. 


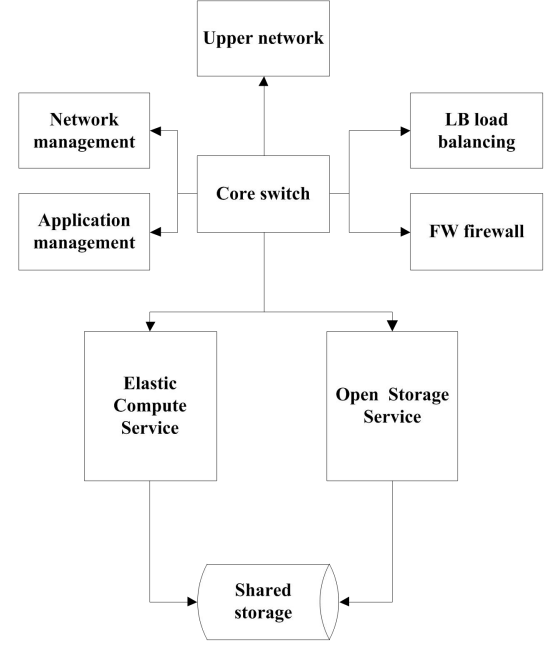

Fig. 6 Structure of the host system of this system

\subsection{System software design}

(1) Acquisition program

The specific process of collection is shown in Figure 7.

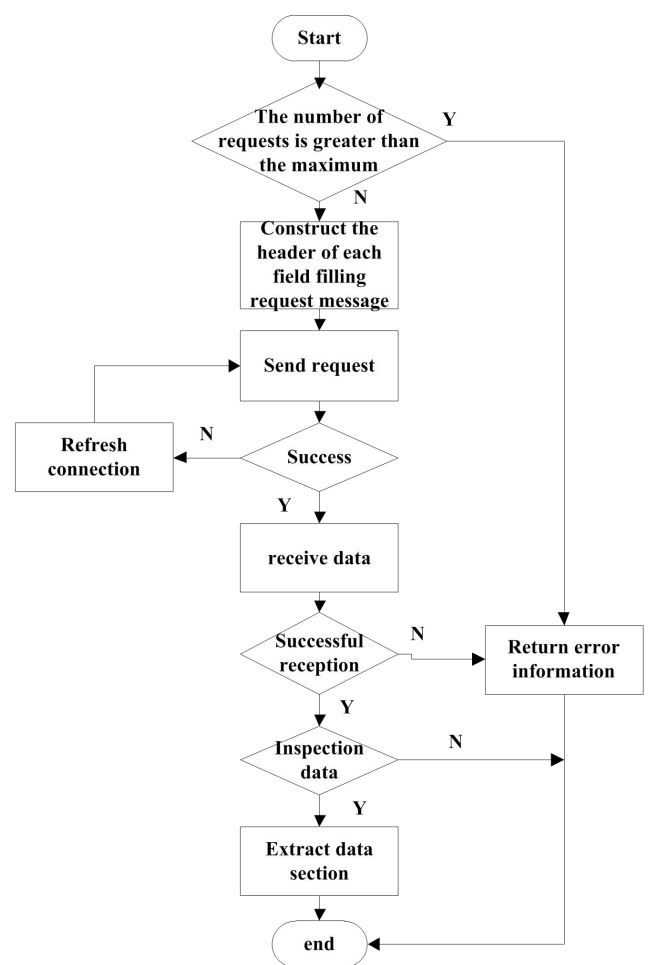

Fig.7 Acquisition program flow 
First, when the device starts running, the acquisition program will use the host as a client to establish a connection with the collector. After the connection is established, the heartbeat packet of the acquisition program will periodically detect the connection status to ensure the reliability of the connection.Secondly, the collection function in the collection program will be called to collect equipment operation data. There are two main collection functions, which are used to correspond to the device register type and the device coil type. Then, the acquisition program will judge the length of the data collected at the same time. Finally, the collected data will be filled into the message in order and transmitted to the host to realize the collection of equipment operation data.

\section{(2) Communication program}

The network access device based on SIM900A establishes a communication connection with the sensor node through the XBEE coordinator. If the XBEE module uses the AT mode, it will lack a lot of information. Here, the API frame mode is used for communication connection. The SIM900A module's GPRS service supports TCP / IP protocol, using TCP / IP protocol to establish network communication [10].

According to the function of sim900a based network access device, the application program of network access device is designed by Arduino software. The specific process is as follows:

Power on the SIM900A-based network access device and perform a series of initialization. Use the XBEE coordinator to establish a GPRS network, search for node devices in the network, and send handshake information \# HELLO \$ to the node. Determine whether the sensor node is connected to the coordinator by receiving feedback information \#RECEIVES.If it is connected, establish a node communication list, otherwise continue testing until a device is connected to the network. Send a AT command to the SIM900A module with a single chip microcomputer to establish a TC P / IP connection with the cloud server. Based on the connection status, determine whether SIM900A has established a good communication connection with the cloud server. If not, apply for a TCP / IP connection again.The single-chip microcomputer waits to receive data. If the serial port connected to SIM900A triggers an interrupt, it means that data is sent from the host server. The single-chip microcomputer analyzes the received command, changes related parameters according to the command, and then looks up the communication list to pass the control command through the XBEE coordinator Send to the sensor node for feedback control operation, the microcontroller continues to wait to receive data. If the serial port connected to the XBEE coordinator triggers an interrupt, it means that data has been uploaded from the sensor node. The microcontroller first analyzes the received data to determine whether the collected data exceeds the set 
threshold. If not, the data is normal. The MCU directly encapsulates the data and uploads it to the cloud server through SIM900A. If the data exceeds the threshold, it indicates that an exception has occurred. The MCU sends an AT instruction to the SIM900A module through the serial port, and sends the exception information to the designated mobile phone. Then send the corresponding feedback instruction to the sensor node through the XBEE coordinator, and then encapsulate the data through the TCP connection of SIM900A, upload it to a specific port on the host server, and realize the data upload.

(3) Host software architecture

The software architecture of this host is shown in Figure 8. Based on the database, the system connects with the network access equipment through the transceiver program on the server, receives the data collected by the sensor node, stores it in the database, and sends the control instruction to the sensor node. The WEB server provides a functional interface to facilitate Users interact with the cloud platform.

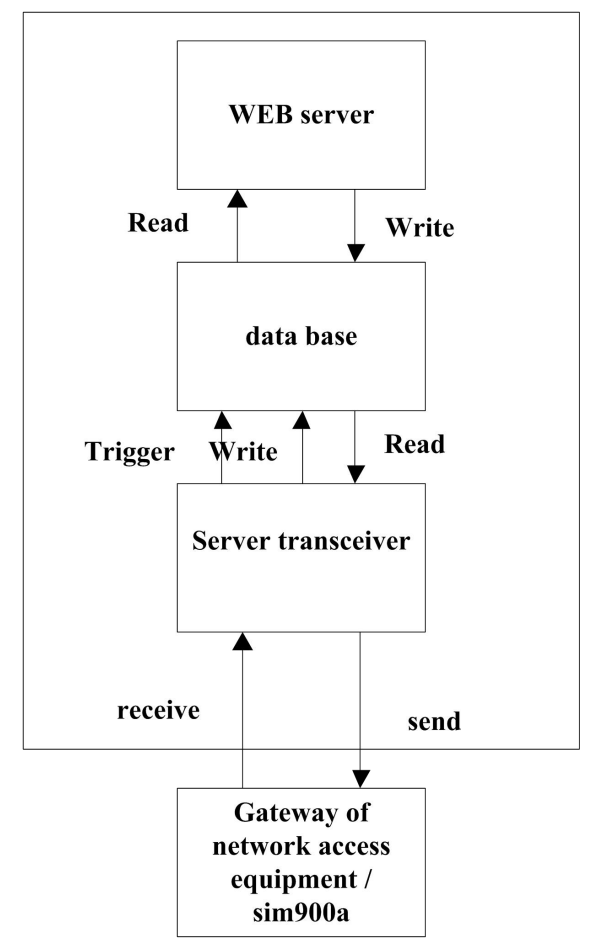

Fig.8 Host software architecture

\section{System simulation test}

This paper designs a remote monitoring system for embedded network equipment faults based on the Internet of things. In order to prove that this system has the characteristics of 
simple structure, easy networking, remote monitoring, cross-platformity, and user-friendly operation, experiments are performed below to verify.

\subsection{Monitoring arrangement}

The experiment monitored four networks located in different rooms. The specific monitoring locations are shown in Figure 9 below.

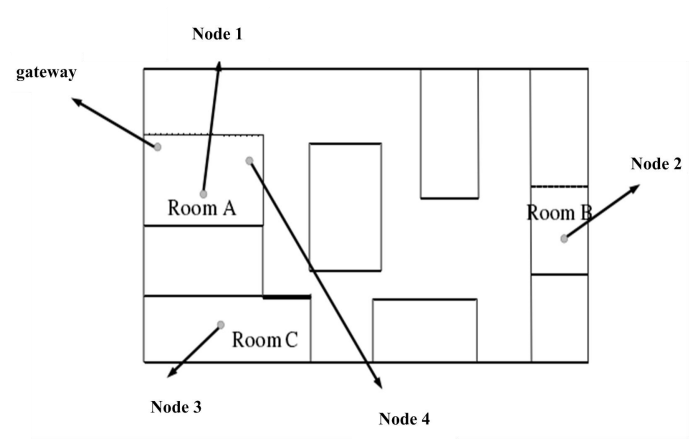

Fig.9 Monitoring arrangement

Nodes 1, 4 and the gateway are placed in Room A on the north side, node 2 is placed in Room B on the south side, and node 3 is placed in Room C. Each node has the same composition and is used to monitor the operating status of the equipment.

\subsection{System operating environment}

This system test is based on the premise that the device is not installed in the network device, according to the operation of the network device, the normal operation and failure of the network device are simulated, and implemented through a variety of network communication methods to achieve the test. The specific test environment is as follows:

Server: Windows Server 2003 32bit;

Client: Windows XP Professional 32bit, Windows7 Ultimate 32bit;

Browser: IE8.0, IE9.0, Google Chrome;

High and low temperature: YSL-GDW-010 high and low temperature test chamber $-50 \sim 80{ }^{\circ} \mathrm{C}$;

Web environment: Wired network, China Unicom $3 \mathrm{G}$ network, mobile $3 \mathrm{G}$ network, telecommunication $3 \mathrm{G}$ network;

Front-end equipment: Hi3515 development board, infrared sensor, level sensor, door status sensor, headset, camera.

\section{3 experiment procedure}

The network equipment monitoring experiment mainly includes four parts: WEB-based application, host, gateway, and lower-level sensor nodes. The collector transmits the collected 
information to the host, and after the initial processing by the microcontroller, it sends it to the X4 gateway through the XBEE module. The gateway forwards the data to the host and stores it in the SQL database.The collector transmits the collected information to the host, and after the initial processing by the microcontroller, it sends it to the $\mathrm{X} 4$ gateway through the XBEE module. The gateway forwards the data to the host and stores it in the SQL database. The control instructions are forwarded to the destination address through the gateway, so as to control the sensor devices and execution devices of the lower computer to achieve the purpose of remote monitoring.

\subsection{Test Results}

(1) System monitoring performance

In order to diagnose the different fault characteristics of the network equipment, four kinds of faults are set on the network equipment, and each kind of fault is deployed according to FIG. 6. The experiment is repeated 10 times.

\begin{tabular}{cc}
\multicolumn{2}{c}{ Table 2 Monitoring accuracy of four monitoring systems } \\
\hline project & Monitoring accuracy (\%) \\
\hline Internet of things & 96.58 \\
web & 92.55 \\
Internert & 88.14 \\
CAN & 90.35 \\
\hline
\end{tabular}

From the experimental results in Table 2, it can be seen that compared with the remote fault monitoring system based on web, Internert, and CAN, the system designed in this paper has higher monitoring accuracy, which shows that the system has higher fault identification capabilities. And robustness, which greatly enhances the effectiveness and accuracy of equipment fault monitoring. (2) Monitoring scope

The monitoring range refers to the maximum range that the monitoring system can cover. Because the monitoring range is circular, it is generally judged by the radius. Table 3 below shows the monitoring ranges of the four monitoring systems.

\begin{tabular}{cc} 
Table 3 Monitoring scope of four monitoring systems \\
\hline project & Monitoring range $(\mathrm{m})$ \\
\hline Internet of things & $\mathbf{1 2 . 3 6}$ \\
web & $\mathbf{8 . 6 9}$ \\
Internert & $\mathbf{9 . 5 8}$ \\
CAN & $\mathbf{1 0 . 2}$
\end{tabular}

From the experimental results in Table 3, it can be seen that the monitoring radius of the system in this paper is not much different from the monitoring radius of the three network 
equipment fault remote monitoring systems based on web, Internert, and CAN. It can be seen that the monitoring scope of this system meets the needs.

\section{Concluding remarks}

Network environment equipment fault diagnosis and protection technology application decision has become an important research topic for network environment operation and maintenance. However, these fault diagnosis systems continue to improve the diagnostic capabilities and expand the scope of diagnosis, but also exposed in the actual operating environment. Some problems occurred, such as excessively high false alarm rates, alarm floods, poor correlation between alarms, and poor monitoring performance of the system itself. In view of the above problems, this paper designs a remote monitoring system for embedded network equipment faults based on the Internet of things. After testing, the system has higher monitoring accuracy and a wider monitoring range, which effectively ensures the stable operation of network equipment. However, the monitoring time is not considered in the research process, which leads to the decrease of remote monitoring efficiency of embedded network equipment fault. Therefore, in the next research, we will focus on the research of monitoring time to improve the efficiency of the system.

\section{Fund projects}

Major Project of the Ministry of Education: "Research on the Construction of Ethnic Disciplines and Majors in Ethnic Areas: Cases of Inner Mongolia and Northeast Ethnic Universities" (number: mjzxzd1405)

\section{References}

[1] Shanmugam, C., E. Chandira, S.:Fault detection in electrical equipment's images by using optimal features with deep learning classifier. Multimedia Tools and Applications, vol. 78,no.19, pp.1-18(2019)

[2] Sun, L. L., Wu, J.H., Jia, H. Q.: Research on fault detection method for heat pump air conditioning system under cold weather. Chinese Journal of Chemical Engineering, vol. 25,no.12, pp. 1812-1819(2017)

[3] Salgado Pilario,K. E., Cao, Y.: Canonical Variate Dissimilarity Analysis for Process Incipient Fault Detection. IEEE Transactions on Industrial Informatics, vol. 14,no.12, pp.5308-5315(2018).

[4] Li, Y., Liu, J., Zhang, W.: Improved multiscale sample entropy and its application in train axle bearing fault detection. Yi Qi Yi Biao Xue Bao/Chinese Journal of Scientific Instrument, vol. 39,no.9, pp.179-186(2018)

[5] Shang, J., Chen, M. Y.: Recursive Dynamic Transformed Component Statistical Analysis for Fault Detection in Dynamic Processes. IEEE Transactions on Industrial Electronics, vol. 65,no.1, pp.578588(2017).

[6] Wu, C. Y., Guo, C.Q., Xie, Z.W.: A Signal-Based Fault Detection and Tolerance Control Method of 
Current Sensor for PMSM Drive. IEEE Transactions on Industrial Electronics, vol. 65,no.12, pp. 96469657(2018)

[7] Faraz, T., Abdul Q. K., Muhammad, A.: Data-Driven Robust Fault Detection and Isolation of Three-Phase Induction Motor. IEEE Transactions on Industrial Electronics, vol. 66,no.6, pp. 4707-4715(2018)

[8] Gao, R., Miao, C., Miao, D.: Correction method of non-uniform illumination image for on-line fault detection of conveyor belt. Zhongguo Kuangye Daxue Xuebao/Journal of China University of Mining and Technology, vol. 47,no.6, pp. 1378-1385(2018)

[9] Ning, Z. K., Yu, J.Y., Pan, Y. N.: Adaptive Event-Triggered Fault Detection for Fuzzy Stochastic Systems With Missing Measurements. IEEE Transactions on Fuzzy Systems, vol. 36,no.4, pp. 22012212(2017)

[10] Huang, D., Yang, X. Y. Chen, S. Y.: A WAMS-based model-free approach of post-fault rotor angle trajectory prediction for on-line transient instability detection. Iet Generation Transmission \& Distribution, vol. 12,no.10, pp.2425-2435(2018) 